\title{
Normalized Adaptive Decision Directed Equalization
}

\author{
A. Lee Swindlehurst, Member, IEEE
}

\begin{abstract}
It has recently been observed that with appropriate stepsize normalization, the convergence speed of the constant modulus (CM) algorithm can be dramatically improved. In this correspondence, it is shown that if a different normalization strategy is used, one that takes into account the finite alphabet structure of the signals, a standard normalized version of the decision directed equalizer (DDE) is achieved. A simulation example is included to demonstrate the faster convergence of the normalized DDE compared with its constant stepsize implementation and the normalized CM.
\end{abstract}

\section{INTRODUCTION}

$\mathbf{T}$ THE SO-CALLED constant modulus (CM) or Godard algorithm [1]-[4] is perhaps the most popular technique for blind adaptive equalization in use today. Its popularity stems mainly from its simple implementation as an LMS adaptive filter, but it suffers from relatively slow convergence, and in certain cases is not guaranteed to converge to a "good" solution. Situations leading to ill convergence have been studied, for example, in [5]. Significant improvements in the convergence rate have recently been observed when the stepsize of the CM algorithm is normalized to force the $a$ posteriori error to zero [6], [7].

An approach often used in conjunction with the CM technique is the so-called decision directed equalizer (DDE). In this approach, the signals must be drawn from a finite alphabet, and the error used to update the LMS adaptive filter is the distance between the current signal estimate and the closest point on the signal constellation. The CM algorithm is typically used to perform an initial equalization, or in other words, to "open the eye" of the signal at the decision points, and then the DDE is switched in to speed convergence. Most of the performance analyses of the DDE have focused on the case where the stepsize is constant, or decreasing to zero (e.g., see [8] and [9]).

In this work, it is shown that a different method of computing the step size in an LMS adaptive equalizer leads to a common normalized version of the DDE. Instead of enforcing a constant modulus constraint, the stepsize is chosen to exploit the structure of the known signal constellation. The performance of the algorithm is illustrated by means of a simple numerical example.

Manuscript received April 15, 1997. This work was supported by the NSF under Grant MIP-9408154. The associate editor coordinating the review of this manuscript and approving it for publication was Prof. K. Buckley.

The author is with the Department of Electrical and Computer Engineering, Brigham Young University, Provo, UT 84602 USA (e-mail: swindle@ee.byu.edu).

Publisher Item Identifier S 1070-9908(98)00664-6.

\section{The Constant Modulus Algorithm}

Suppose a complex-valued $m$-vector $\mathbf{x}(t)$ is observed at the time samples $t=1, \cdots, N$. The vector $\mathbf{x}(t)$ could represent $m$ consecutive samples of a single channel time series, or a snapshot from an array with $m$ elements, or a combination of both. The goal is to design an $m$-vector of filter coefficients $\mathbf{w}(t)$ such that the filtered signal, $y(t)=\mathbf{w}^{*}(t) \mathbf{x}(t)$, has constant (e.g., unity) modulus for all $t$. Here, the symbol $\{\cdot\}^{*}$ denotes a conjugate transpose. In the standard CM approach, $\mathbf{w}(t)$ is estimated by defining the error signal $\epsilon_{\mathrm{CM}}(t)=$ $|y(t)|^{2}-1$, and using the LMS adaptive algorithm to minimize $\epsilon_{\mathrm{CM}}^{2}(t)$. The weight update is given by

$$
\begin{aligned}
\mathbf{w}(t+1) & =\mathbf{w}(t)-\mu^{\prime}(t) \frac{\partial \epsilon_{\mathrm{CM}}^{2}(t)}{\partial \mathbf{w}} \\
& =\mathbf{w}(t)-\mu(t) \bar{y}(t) \epsilon_{\mathrm{CM}}(t) \mathbf{x}(t)
\end{aligned}
$$

where $\bar{y}(t)$ is the conjugate of $y(t)$, and where all constant terms have been absorbed into the step length $\mu(t)$.

While guidelines exist for choosing $\mu(t)$, a convenient technique is to calculate $\mu(t)$ such that the a posteriori error is zero; that is, choose $\mu(t)$ such that

$$
\left|\mathbf{w}^{*}(t+1) \mathbf{x}(t)\right|^{2}=1 \text {. }
$$

This results in the so-called normalized CM (NCM) algorithm, and has been empirically demonstrated to achieve much faster convergence than constant step size implementations [6], [7]. The value of $\mu(t)$ that satisfies (3) is easily shown to be

$$
\mu(t)=\frac{|y(t)|-1}{|y(t)| \epsilon_{C M}(t)|| \mathbf{x}(t)||^{2}} .
$$

It is important to note that, once the step length is chosen in this way, the specification of an error function such as $\epsilon_{C M}^{2}(t)$ is no longer needed. With the normalization of (4), any error criterion will result in the same update for $\mathbf{w}(t)$. In fact, the problem could simply be posed as that of finding $\tilde{\mu}(t)$ such that

$$
\mathbf{w}(t+1)=\mathbf{w}(t)-\tilde{\mu}(t) \mathbf{x}(t)
$$

satisfies (3) without even introducing an error term.

\section{DECISION DIRECTED EQUALIZATION}

Suppose the desired signal transmitted through the channel is drawn from a finite alphabet of $P$ symbols, and is sampled at the symbol rate; i.e., $s(t)=\alpha_{k}, k=0, \cdots, P-1$. In the DDE approach, the weight vector is adapted in a manner similar to that above, as follows:

$$
\mathbf{w}(t+1)=\mathbf{w}(t)-\mu(t) \bar{\epsilon}_{\mathrm{DD}}(t) \mathbf{x}(t)
$$


but in this case the error is defined by

$$
\begin{aligned}
\hat{p} & =\arg \min _{p}\left|y(t)-\alpha_{p}\right| \\
\epsilon_{\mathrm{DD}}(t) & =y(t)-\alpha_{\hat{p}}
\end{aligned}
$$

which is simply the residual remaining after a symbol decision is made using $y(t)$. As with all such adaptive filters, the stepsize controls the rate of convergence; to avoid instability, the most common approach is to choose $\mu(t)$ to be a small constant. However, this is a fairly conservative approach, and consequently the convergence may be rather slow.

One advantage of the DDE over the CM equalizer is its ability to converge to a "good" solution in cases where CM cannot. As an illustration of this fact, consider the problem of separating two BPSK signals received by an antenna array. Suppose

$$
\mathbf{x}(t)=\mathbf{a}_{1} s_{1}(t)+\mathbf{a}_{2} s_{2}(t)
$$

where $\mathbf{a}_{k}$ is the array response (spatial signature) for signal $k$, and $s_{k}(t)= \pm 1$. Perfect spatial equalization occurs, for example, if either $\mathbf{w}^{*} \mathbf{a}_{1}=0$ and $\mathbf{w}^{*} \mathbf{a}_{2}=1$, or $\mathbf{w}^{*} \mathbf{a}_{1}=1$ and $\mathbf{w}^{*} \mathbf{a}_{2}=0$, and both solutions satisfy the CM constraint $|y(t)|^{2}=1$. However, any $\mathbf{w}$ that satisfies

$$
\mathbf{w}^{*}\left[\begin{array}{ll}
\mathbf{a}_{1} & \mathbf{a}_{2}
\end{array}\right]=\left[\begin{array}{ll}
\sqrt{1-\alpha^{2}} & j \alpha
\end{array}\right] e^{j \phi}
$$

for arbitrary real $\phi$ and $0<\alpha<1$ also satisfies the constraint, but clearly does not provide perfect equalization. While the two signals could still be separated from $\mathbf{w}^{*} \mathbf{x}(t)$ since they are in quadrature, additional processing would be necessary to detect this situation and remedy it. Such an undesirable solution is not possible with the DDE.

To make the convergence of the DDE more rapid, a normalized stepsize could be used. For example, one could force the a posteriori CM error to zero as in (3), but as explained above the resulting DDE would be identical to the standard NCM approach. Instead, consider the general form for the weight update given in (5), and choose $\tilde{\mu}(t)$ as the smallest (in absolute value) of the $P$ solutions to the equations

$$
\mathrm{w}^{*}(t+1) \mathrm{x}(t)=\alpha_{p}, \quad p=0,1, \cdots, P-1 .
$$

Note that the resulting solution for $\tilde{\mu}(t)$ will in general be complex. As with the NCM approach, once the step size is constrained in this way, the definition of an error function $\epsilon(t)$ is superfluous.

Solving (9) for $\tilde{\mu}(t)$ leads to the following expression:

$$
\tilde{\mu}(t)=\frac{\bar{y}(t)-\bar{\alpha}_{\hat{p}}}{\|\mathbf{x}(t)\|^{2}}
$$

where $\hat{p}$ is the symbol decision defined by (7). The interesting point to make here is that if (10) is substituted into (5), the resulting algorithm is identical to the DDE if the DDE stepsize is chosen as

$$
\mu(t)=\|\mathbf{x}(t)\|^{-2} .
$$

For this reason, the weight update of (6) implemented with the step size in (11) is referred to here as the normalized DDE (NDDE). Normalizing the step length as in (11) is

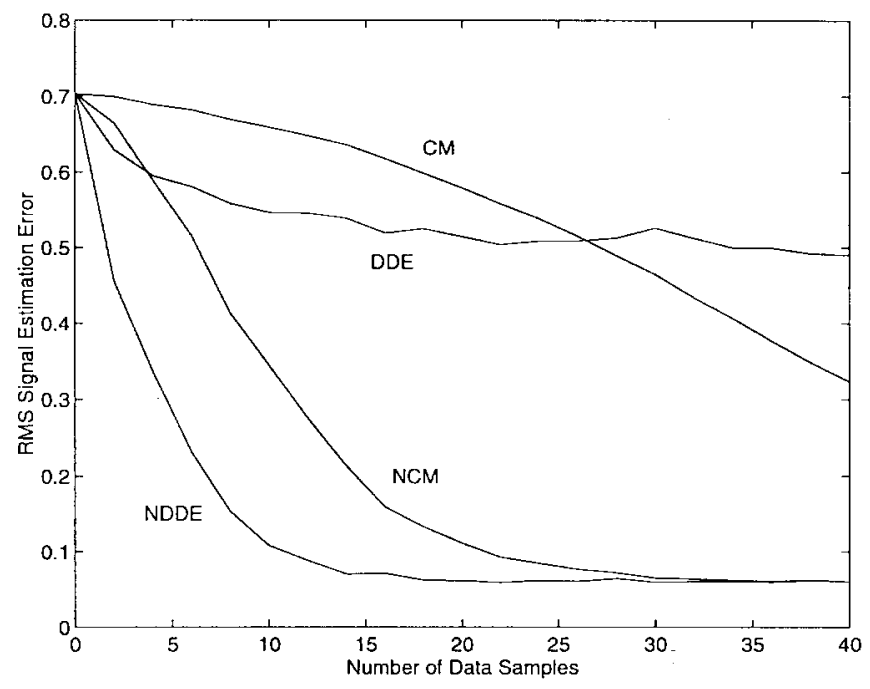

Fig. 1. Convergence of several blind adaptive equalizers.

a common technique used in LMS adaptive equalizers to make the convergence of the algorithm independent of the power of the signal $\mathbf{x}(t)$. What is interesting here is that this normalization also results from constraining the a posteriori error to zero.

With fractionally sampled (FS) data, the DDE and NDDE approaches are implemented in the same way as the FS-CM algorithm (e.g., see [10]), where a separate filter is adapted at the symbol rate for each of the fractional subchannels. The stepsize is the same for each subchannel, and is normalized based on the sum of all the subchannel filter outputs. Whether or not fractionally sampled data is involved, both the DDE and the NDDE approach presented here require that the symbol timing of the desired signal be known a priori. The standard $\mathrm{CM}$ approach also implicitly relies on this assumption, since with pulse shaping a CM digital signal is constant modulus only at the symbol center (provided the pulse has been designed so there is no intersymbol interference). However, both algorithms are quite robust in this regard.

\section{A NumeriCAL EXAMPLE}

A simulation was conducted in which two synchronous symbol-rate QPSK signals were received by a three element uniform linear array with half wavelength spacing. The directions of arrival of the two signals were $5^{\circ}$ and $25^{\circ}$, and both were generated with a random phase and a signal-to-noise ratio (SNR) of $25 \mathrm{~dB}$. The equivalent signal-to-interferenceplus-noise ratio is slightly less than $0 \mathrm{~dB}$. Fig. 1 shows a plot of the root mean square (RMS) error (averaged over 100 trials) of the various approaches described above versus the number of samples used to adapt the spatial filter weights. The values of the stepsize for the CM and DDE algorithms were chosen to give the fastest possible convergence; for this example the resulting values were $\mu=0.012$ and 0.08 , respectively. The initial weight vector was chosen to be $\mathbf{w}=\left[\begin{array}{lll}1 & 0 & 0\end{array}\right]^{T}$, and the RMS error was computed based on whichever of the two signals the algorithms converged to. 
In this example, the NDDE converges nearly three times faster than NCM, and an order of magnitude faster than the constant step size CM approach. The standard DDE converges quickly at first, but is unable to fully eliminate the interferer. It should be noted that, in many of the cases studied, the difference in performance between NDDE and NCM was smaller than for this example, although NDDE consistently converged more quickly.

\section{REFERENCES}

[1] D. N. Godard, "Self-recovering equalization and carrier tracking in twodimensional data communication systems," IEEE Trans. Commun., vol. COMM-28, pp. 1867-1875, Nov. 1980.

[2] J. Treichler and B. Agee, "A new approach to multipath correction of constant modulus signals," IEEE Trans. Acoust., Speech, Signal Processing, vol. ASSP-31, pp. 459-472, Apr. 1983.
[3] J. Treichler and M. Larimore, "New processing techniques based on the constant modulus adaptive algorithm," IEEE Trans. Acoust., Speech, Signal Processing, vol. ASSP-33, pp. 420-431, Apr. 1985.

[4] R. Gooch and J. Lundel, "The CM array: An adaptive beamformer for constant modulus signals," in Proc. IEEE ICASSP, 1986, pp. 2523-2526.

[5] Z. Ding, R. Kennedy, B. Anderson, and C. Johnson, "Ill-convergence of Godard blind equalizers in data communication systems," IEEE Trans. Commun., vol. 39, pp. 1313-1327, Sept. 1991.

[6] K. Hilal and P. Duhamel, "A convergence study of the constant modulus algorithm leading to a normalized-CMA and a block-normalized CMA," in Proc. EUSIPCO, 1992.

[7] D. Jones, "A normalized constant modulus algorithm," in Proc. ICASSP, Atlanta, GA, 1996, pp. 694-697.

[8] J. E. Mazo, "Analysis of decision directed equalizer convergence," Bell Syst. Tech. J., vol. 59, pp. 1857-1876, Dec. 1980.

[9] O. Macchi and E. Eweda, "Convergence analysis of self-adaptive equalizers," IEEE Trans. Inform. Theory, vol. IT-30, pp. 161-174, Mar. 1984.

[10] Y. Li and Z. Ding, "Global convergence of fractionally spaced Godard (CMA) adaptive equalizers," IEEE Trans. Signal Processing, vol. 44, pp. 818-826, Apr. 1996. 